\title{
The Effects of Roughness on Adhesion Hysteresis
}

\author{
Zheng Wei ${ }^{\text {a }}$, Meng-Fu He ${ }^{\text {a }}$ and Ya-Pu Zhao ${ }^{\text {b,* }}$ \\ ${ }^{a}$ College of Mechanical and Electrical Engineering, Beijing University of Chemical Technology, \\ Beijing 100029, People's Republic of China \\ ${ }^{\mathrm{b}}$ State Key Laboratory of Nonlinear Mechanics, Institute of Mechanics, \\ Chinese Academy of Sciences, Beijing 100080, People's Republic of China
}

Received in final form 5 January 2009; revised 31 October 2009; accepted 31 October 2009

\begin{abstract}
Adhesion hysteresis is defined as the difference between the work needed to separate two surfaces and that originally gained on bringing them together. Adhesion hysteresis is a common phenomenon in most surface/interface interactions. This paper studies the effects of surface roughness on adhesion hysteresis. We assumed that the surface asperity height distribution is Gaussian. Numerical simulations based on Fuller's model showed that adhesion hysteresis depended upon a single dimensionless parameter, the adhesion parameter, which represents the statistical average of a competition between the compressive forces exerted by the higher asperities, which are trying to separate the surfaces, and the adhesion forces of the lower asperities which are trying to hold the surfaces together.
\end{abstract}

(c) Koninklijke Brill NV, Leiden, 2010

Keywords

Adhesion hysteresis, surface roughness, adhesion parameter

\section{Introduction}

Adhesion plays a significant role in microelectromechanical systems (MEMS), scanning probe microscope (SPM) measurements, nanomanipulation, nanotribology, nanowear and related fields. Due to the very large surface-to-volume ratio, adhesion is one of the major factors that limits the widespread use of microelectromechanical systems, and thus attracts much attention in the MEMS community [1-3].

Adhesion hysteresis is by definition the difference between the work needed to separate two surfaces or molecules and the work gained in bringing them together $[4,5]$. The former is generally greater than the latter. Dutroski [6] first observed hysteresis behavior in contact deformation. This phenomenon has also been reported

\footnotetext{
* To whom correspondence should be addressed. Fax: +86-10-6256-1284; e-mail: yzhao@imech.ac.cn 
by Israelachvili and his colleagues [5]. They published a number of papers on the adhesion between thin layers and in their work used of a surface force apparatus (SFA), [5, 7-10]. Their results were startling at first, because they reported the existence of adhesion hysteresis. According to the tenets of thermodynamics the work of adhesion represents a change in free energy between two defined equilibrium states, and the properties of such states depend on the states themselves and not on how they are approached [11]. In the case of solid-solid contacts, the hysteresis has generally been attributed to viscoelastic bulk deformations of the contacting materials or to plastic deformations of locally contacting asperities [12-16]. In the case of solid-liquid contacts, hysteresis has usually been attributed to surface roughness or to chemical heterogeneity [17], but such explanations do not apply to molecularly smooth chemically homogeneous surface because even in these cases significant hysteresis does exist [18]. Israelachvili believes there are two other sorts of hysteresis inherent in many adhesion situations: mechanical and chemical hysteresis [5]. Mechanical hysteresis arises from mechanical instabilities and chemical hysteresis from inter-diffusion, reorientation of polar molecules, or the exchange of chemical species from bulk to surface. What distinguishes chemical hysteresis from mechanical hysteresis is that during chemical hysteresis the chemical groups at the surfaces are different on separation, from what they were when the surfaces were about to be joined [5].

Apart from the above mentioned factors, adhesion hysteresis may also arise from physical bonding [19]. It is known that many surfaces contain defects such as dangling chains. These chains can act as connector molecules resulting in high adhesion energies [20, 21]. Adhesion hysteresis, between polydimethylsiloxane elastomers, have been observed on model networks with differing amounts of dangling chains, using the Johnson, Kendall and Roberts (JKR) test. The adhesion hysteresis was found to increase with increasing amounts of dangling chains. It is believed that this hysteresis arises from the inter-diffusion of chains across the interface [19]. Other papers have attributed the phenomenon of adhesion hysteresis to: surface rearrangement at the polymer interface [22]; the surface energy and phase state of the substrate [23]; capillary forces due to ambient humidity [7]; possible inter-digitation of the polymer across the interface [10]; and even to chemical reactions occurring at the interface [24].

Attard and Parker claimed that they found two types of hysteresis [25]. First, the position at which the bodies jumped into contact was smaller than that at which they jumped apart. We think this type of adhesion hysteresis can be classified as an Israelachvili's mechanical hysteresis [5]. This phenomenon, which has also been found by simulations $[26,27]$ is due in part to the finite range of the van der Waals interaction and obviously cannot be predicted by JKR or other contact theories. The same phenomenon may be found in the force curve of atomic force microscopy. Second, it was found that the deformation-loading curve did not coincide with the deformation-unloading curve even when both corresponded to contact and that the 
maximum tension on unloading, which is the negative of the pull-off force, depended upon the value of the maximum applied load.

Although much effort has been made, the mechanism of the adhesion hysteresis is still not well understood and as seen above, a number of factors can be responsible for adhesion hysteresis. To our knowledge, the effect of roughness on adhesion hysteresis has not been adequately investigated. In this paper, we attempted to study this type of adhesion hysteresis by varying the topography of contacting surfaces.

\section{The Model of Surface Roughness}

It has long been realized that surfaces are rough on a microscopic or nanoscopic scale, and this causes the real area of contact to be extremely small compared to the nominal area. The theory of elastic contact of spheres was first formulated by Hertz [28] and later modified by Johnson, Kendall and Roberts, using adhesive forces [29]. The problem of contact between two planar rough surfaces had been analyzed by Greenwood and Williamson [30]. The influence of surface roughness on the adhesion between rubber (or any other elastic solid) and a hard substrate, has been given in a classic paper by Fuller and Tabor [31]. Since then a great deal of progress has been made in this area. The effect of surface roughness on adhesion at the contact of rough solids has been studied analytically in great detail by Chang et al. [32] and Chowdhury and Ghosh [33] using Greenwood's rough surface model [30]. In a recent study, Zhang and Zhao [34] developed a theoretical model to describe the adhesion between plastic deformable fractal surfaces whose asperity heights conform to a general distribution. In this work, expressions for real contact areas, total loading and the required separation force, were obtained [34]. In general, asperities were found to occur at different lengths and the surface topography was found to be a non-stationary random process [35]. The concepts in fractal geometry were considered to be a better alternative than the use of the Greenwood's random rough surface model [36, 37]. However, as this paper deals with the effect of roughness on adhesion hysteresis, a simple roughness model was adopted and the JKR solution model, usually used to describe the adhesive contact between nominally flat elastic bodies, was modified to take into account the roughness of the surface.

We will consider the contact of an elastic rough surface with an ideal flat rigid surface (as shown in Fig. 1). The surface roughness was treated as a random series of asperities with a Gaussian height distribution of:

$$
\phi(z)=\frac{1}{\sqrt{2 \pi} \sigma} \exp \left(-\frac{z^{2}}{2 \sigma^{2}}\right),
$$

where $\phi(z) \mathrm{d} z$ is the probability that an asperity has a height between $z$ and $z+\mathrm{d} z$ above the plane defined by the mean asperity height. The asperities are all assumed to have spherical caps of the same constant radius $R$ and a Gaussian height distribution of standard deviation $\sigma$. So the distribution function satisfies:

$$
\int_{-\infty}^{\infty} \phi(z) \mathrm{d} z=1
$$




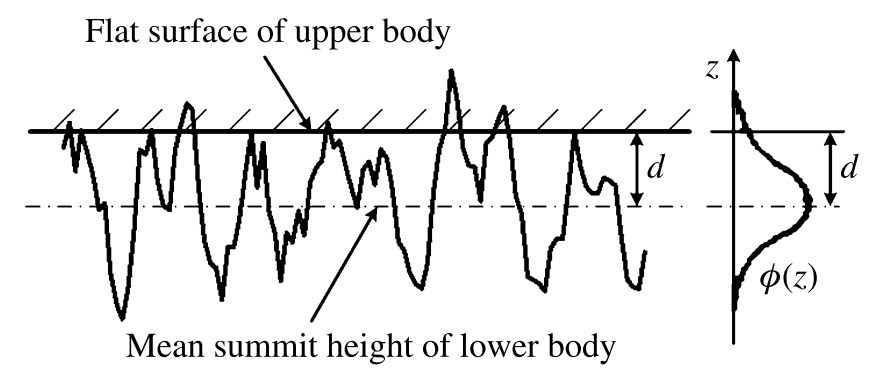

Figure 1. Contact of elastic rough surface with ideal flat rigid surface.

If there are $N$ asperities in the rough surface, the expected number of rough contacts with the real flat rigid surface will be:

$$
n=N \int_{d}^{\infty} \phi(z) \mathrm{d} z .
$$

\section{JKR Contact Model}

Firstly, it is necessary to analyze the relation between the force $P$ to compress a single spherical asperity, and the displacement $\delta$ relative to the undeformed part of the plane, before we start the problem of a rough elastic surface with an ideal flat rigid surface.

If an elastic sphere of radius $R$ is pressed under a load $P$ against a rigid plane, the radius of contact $a$ and the elastic displacement $\delta$ are given by [38]:

$$
\begin{gathered}
\delta=\frac{a^{2}}{3 R}+\frac{2 P}{3 a K}=\frac{1}{3 K^{2 / 3} R^{1 / 3}} \frac{P_{1}+2 P}{P_{1}^{1 / 3}}, \\
P=\frac{a^{3} K}{R}-\sqrt{6 \pi a^{3} K w_{\mathrm{a}}}=P_{1}-\sqrt{6 \pi w_{\mathrm{a}} R P_{1}},
\end{gathered}
$$

with $K=\frac{4}{3} E^{*}=\frac{4}{3} \frac{1-v^{2}}{E}, P_{1}=\frac{a^{3} K}{R}$, where $v$ is Poisson's ratio and $E$ is the Young's modulus, and $w_{\mathrm{a}}$ is the work of adhesion.

The limits of stability at fixed load and at fixed grips, respectively, are $P_{\mathrm{c}}=$ $\frac{3}{2} \pi R w_{\mathrm{a}}, \delta_{\mathrm{c}}=\left(\frac{3}{4} \frac{\pi^{2} R w_{\mathrm{a}}^{2}}{K^{2}}\right)$. Eliminating $P_{1}$ between the two equations (4) and (5), one obtains the two expressions:

$$
\begin{gathered}
\frac{\delta}{\delta_{\mathrm{c}}}=(3 \chi-1)\left(\frac{\chi+1}{9}\right)^{1 / 3}, \quad \chi \geqslant 0, \\
\frac{\delta}{\delta_{\mathrm{c}}}=-(3 \chi+1)\left(\frac{1-\chi}{9}\right)^{1 / 3}, \quad 0 \leqslant \chi \leqslant \frac{2}{3},
\end{gathered}
$$

where $\chi=\sqrt{1+\frac{P}{P_{\mathrm{c}}}}$. The functions have been plotted in Fig. 2, where the branch bc corresponds to equation (7). The maximum force which can be sustained by 


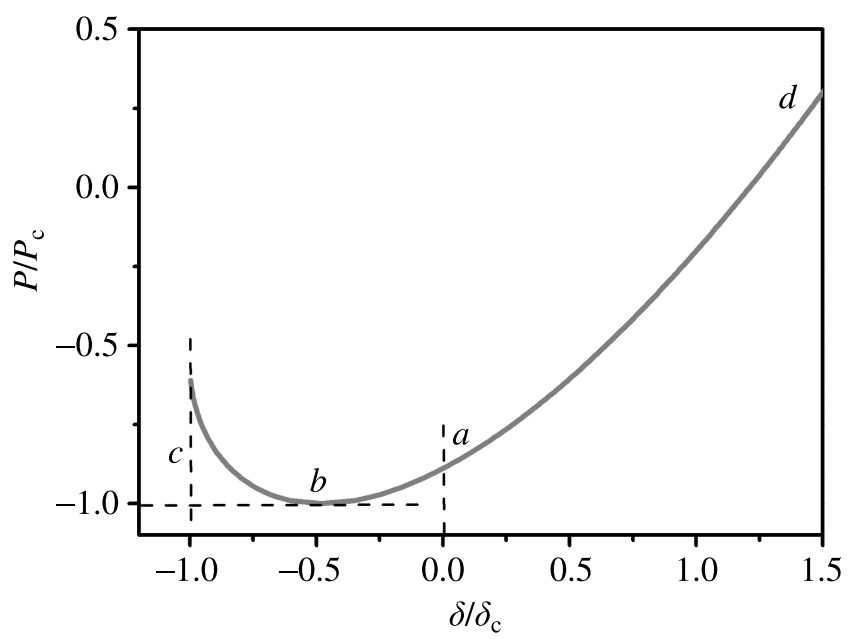

Figure 2. The relation between load and penetration in the JKR model.

adhesion before separation is $P_{\mathrm{c}}$, while $\delta_{\mathrm{c}}$ represents the maximum extension of the asperity above its undeformed height, before separation occurs.

Hence from the Fig. 2 we obtain the relation:

$$
\frac{P}{P_{\mathrm{c}}}=F\left(\frac{\delta}{\delta_{\mathrm{c}}}\right)
$$

Hysteresis can be seen in Fig. 2. For an individual asperity contact with an elastic plane, the curve $a d$ represents the loading path while the curve dabc represents the unloading path. If we consider starting from point $d$, we see that the load decreases regularly, but cannot go beyond point $b$. On the other hand if one regularly decreases the penetration, one cannot go beyond point $c$. These are the two instability points in the equilibrium curve.

\section{Adhesion Hysteresis Due to Roughness}

In the loading process, the total contact force per unit area between the planes is the sum of the forces exerted by all the asperities whose height exceeds $d$, i.e:

$$
\begin{aligned}
P_{\text {load }} & =N \int_{d}^{\infty} P(z) \phi(z) \mathrm{d} z=\frac{N P_{\mathrm{c}}}{\sigma \sqrt{2 \pi}} \int F\left(\frac{\delta}{\delta_{\mathrm{c}}}\right) \exp \left(-\frac{z^{2}}{2 \sigma^{2}}\right) \mathrm{d} z \\
& =\frac{N P_{\mathrm{c}}}{\sqrt{2 \pi}} \int_{0}^{\infty} F\left(\frac{\Delta}{\Delta_{\mathrm{c}}}\right) \exp \left(-\frac{1}{2}(h+\Delta)^{2}\right) \mathrm{d} \Delta,
\end{aligned}
$$

where $\delta=z-d, \Delta=\delta / \sigma, \Delta_{\mathrm{c}}=\delta_{\mathrm{c}} / \sigma, h=d / \sigma$.

However, when the planes are first compressed and then separated, a large number of asperities come into contact and are then elongated to a critical value $\delta_{\mathrm{c}}$ at 


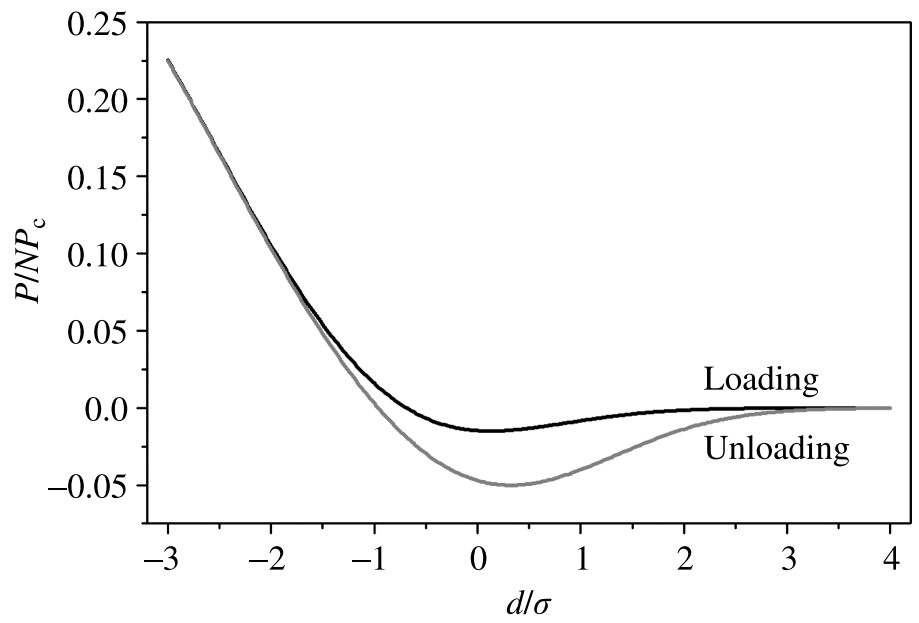

Figure 3. Adhesion hysteresis due to surface roughness $1 / \Delta_{\mathrm{c}}=\sigma / \delta_{\mathrm{c}}=1$. The loading path and unloading path compose a loop.

which point the contact is broken. Asperities with a height $z>d$ are compressed, those with height $d-\delta_{\mathrm{c}}<d<d$ are stretched. This implies that all asperities of height $z>d-\delta_{\mathrm{c}}$ are in contact [14]. Hence the total forces exerted by the contacted asperities are given by:

$$
P_{\text {unload }}=\frac{N P_{\mathrm{c}}}{\sqrt{2 \pi}} \int_{-\Delta_{\mathrm{c}}}^{\infty} F\left(\frac{\Delta}{\Delta_{\mathrm{c}}}\right) \exp \left(-\frac{1}{2}(h+\Delta)^{2}\right) \mathrm{d} \Delta .
$$

Equations (9) and (10) suggest that there is some difference between the loading and unloading processes, which essentially represents the adhesion hysteresis due to surface roughness. Denoting $1 / \Delta_{c}=\sigma / \delta_{c}=1$, the variation of the normalized pull-off force with the normalized penetration has been plotted in Fig. 3. It can be seen that the two branches are quite distinct and that hysteresis is obviously represented within the figure.

Figure 4 shows the curve of force-displacement hysteresis for various values of a dimensionless parameter, $1 / \Delta_{\mathrm{c}}=\sigma / \delta_{\mathrm{c}}$. The energy dissipated during the loadingunloading cycle is given by the area enclosed by the loading path and unloading path. This area corresponds to adhesion hysteresis. These plots clearly depict the effect of the dimensionless parameter on adhesion hysteresis.

To see the effect of this dimensionless parameter more precisely, let us examine it in somewhat greater detail. The curve giving the dissipation energy as a function of the dimensionless parameter is shown in Fig. 5. When the dimensionless parameter $1 / \Delta_{c}$ is increased, the elastic surface is changes so that it becomes rougher and the dissipation energy decreases and tends to zero. From Fig. 4 it can see that when the dimensionless parameter $1 / \Delta_{c}$ is increased, the adhesion force becomes very small. 

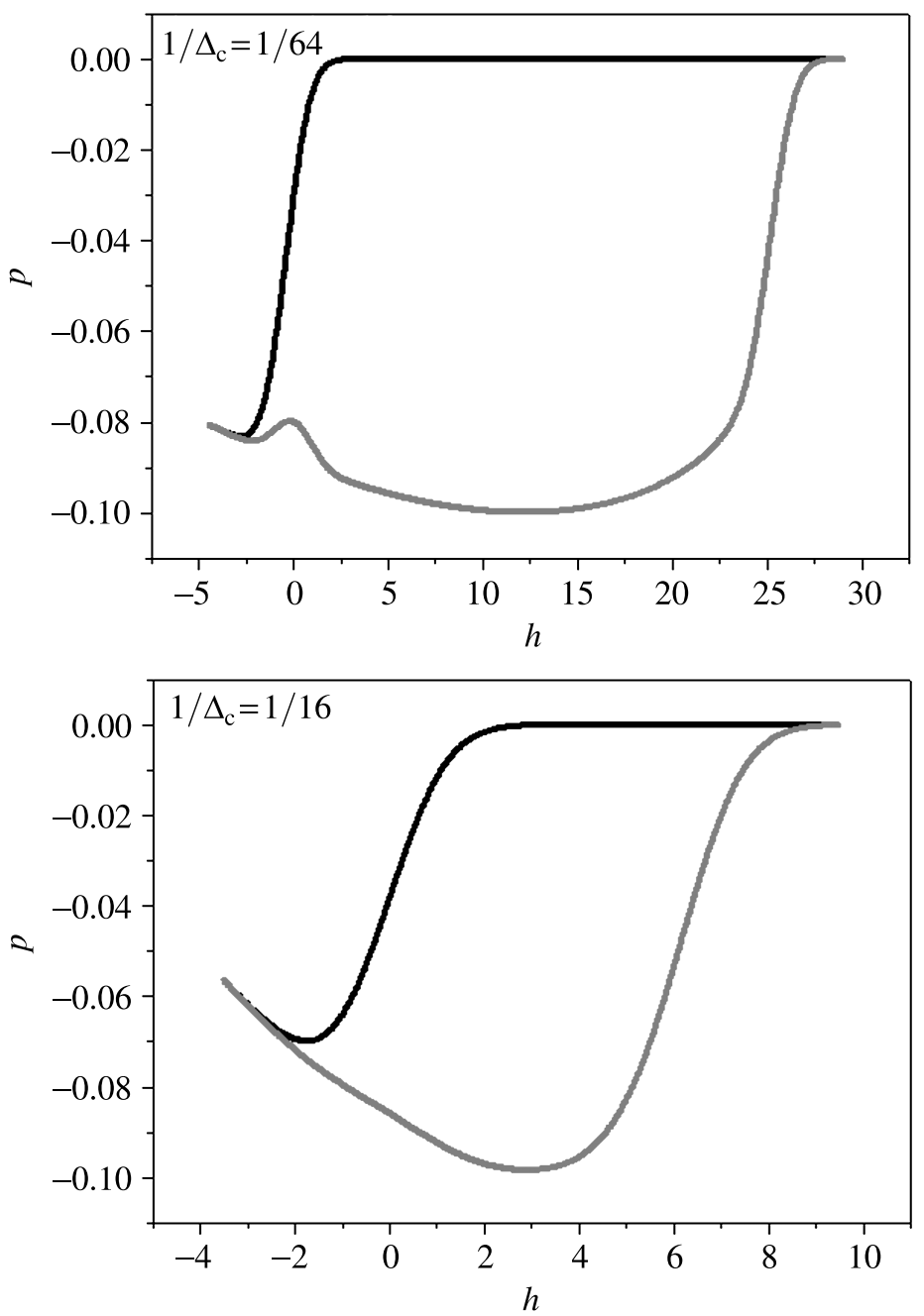

Figure 4. Elastic contact of a rough surface on a flat rigid plane. Normalized force $p=P / N P_{\mathrm{c}} v s$ the normalized distance $h=d / \sigma$ for various values of the parameter $\sigma / \delta_{\mathrm{c}}$.

It is important to discuss the physical implications of the dimensionless parameters $\sigma / \delta_{\mathrm{c}}$ on which the adhesion hysteresis depends. We may conveniently call this quantity the 'adhesion parameter' and it can be written more explicitly as [31]:

$$
\frac{1}{\Delta_{\mathrm{c}}}=\frac{\sigma}{\delta_{\mathrm{c}}}=3 \sigma\left(\frac{2 K}{9 \pi R^{1 / 2} w_{\mathrm{a}}}\right)^{2 / 3} .
$$

If we consider $\left(1 / \Delta_{c}\right)^{3 / 2}$ and omit the numerical terms we obtain a modified adhesion parameter, which we may call $\theta$, given by:

$$
\theta=\frac{E \sigma^{3 / 2}}{R^{1 / 2} w_{\mathrm{a}}}=\frac{E \sigma^{3 / 2} R^{1 / 2}}{R w_{\mathrm{a}}} .
$$



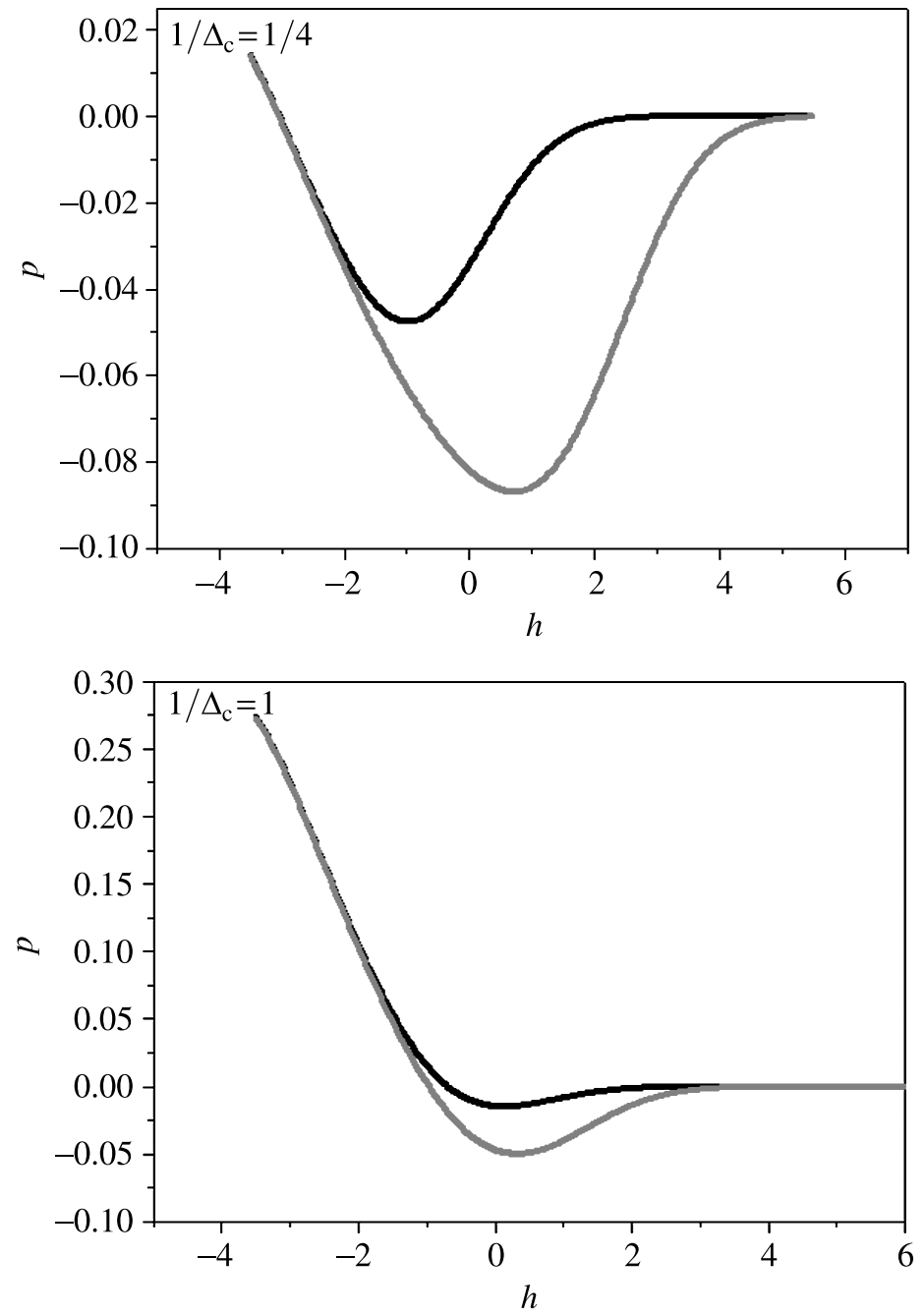

Figure 4. (Continued.)

We see that the denominator of equation (12) is a measure of the adhesive force experienced by a sphere of radius $R$. The numerator turns out to be the elastic force required to push a sphere of radius $R$ to a depth of $\sigma$ into an elastic solid of modulus $E$. Clearly these adhesion parameters represents the statistical average of a competition between the compressive forces exerted by the higher asperities which are trying to separate the surfaces and the adhesion forces between the lower asperities which are trying to hold the surfaces together. When the adhesion parameter is small, the adhesive factor dominates and the contact surfaces are quite smooth, the adhesion hysteresis is high. The extreme case is $\theta \ll 1$, which corresponds to same height asperities in contact with a flat surface and the adhesion hysterersis achieves its maximum. As the adhesion parameter increases, the surface roughness 


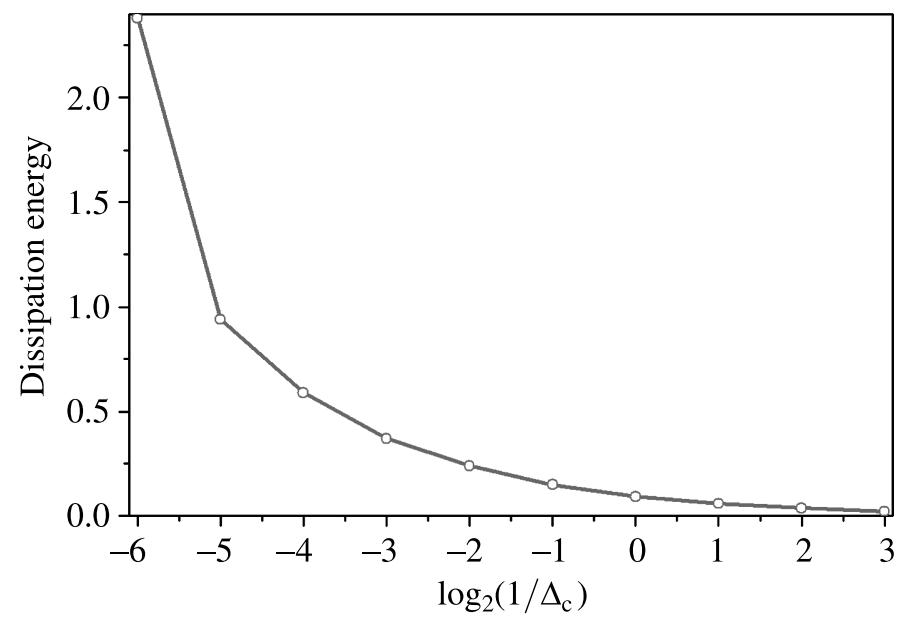

Figure 5. Adhesion hysteresis due to surface roughness for various values of the parameter $\sigma / \delta_{\mathrm{c}}$.

increases, the adhesive factor decreases, and the adhesion hysteresis is low. The extreme case occurs when $\theta \gg 1$, which corresponds to an elastic contact which does not involve adhesion. In this case the adhesion hysteresis is zero.

\section{Conclusion}

This paper has been concerned with the effect of surface roughness on the adhesion hysteresis. Using the Fuller and Tabor analysis [31], the loading and unloading path was obtained. It was found that adhesion hysteresis was determined by an adhesion parameter which is defined by the ratio between the dispersion of asperity heights and the elastic extension which an asperity can sustain before it breaks contact with the other surface. When the adhesion parameter increases, the elastic surface becomes rougher, and the adhesion hysteresis decreases and tends to zero.

\section{Acknowledgements}

This research has been financially supported by the National Basic Research Program of China (973 Program, Grant No. 2007CB310500) and the National Natural Science Foundation of China (NSFC, Grant Nos 10772180 and 10721202) and the Young Scholars Fund of Beijing University of Chemical Technology (Grant No. QN0716).

\section{References}

1. R. P. Feynman, J. Microelectromech. Syst. 1, 60 (1992).

2. Y.-P. Zhao, L. S. Wang and T. X. Yu, J. Adhesion Sci. Technol. 17, 519 (2003).

3. R. Maboudian, Surface Sci. Reports 30, 207 (1998).

4. N. Maeda, Y.-L. Chen, M. Tirrell and J. N. Israelachvili, Science 297, 379 (2002). 
5. Y.-L. Chen, C. A. Helm and J. N. Israelachvili, The Journal of Physical Chemistry 95, 10736 (1991).

6. R. C. Dutroski, Trans. ASME J. Lubr. Technol. 91F, 732 (1969).

7. H. Yoshizawa, Y.-L. Chen and J. N. Israelachvili, J. Phys. Chem. 97, 4128 (1993).

8. H. Yoshizawa, Y.-L. Chen and J. N. Israeiachvili, Wear 168, 161 (1993).

9. H. Yoshizawa and J. N. Israeiachvili, Thin Solid Films 246, 71 (1994).

10. J. N. Israelachvili, Y.-L. Chen and H. Yoshizawa, J. Adhesion Sci. Technol. 8, 1231 (1994).

11. G. N. Lewisand and M. Randall, in: Thermodynamics, K. S. Pitzer and L. Brewer (Eds), 2nd edn. McGraw-Hill, New York, NY (1961).

12. F. P. Bowden and D. Tabor, Friction and Lubricution. Methuen, London (1967).

13. J. A. Greenwood and K. L. Johnson, Philos. Mag. A. 43, 697 (1981).

14. P. Attard, Phys. Rev. E 63, 061604 (2001).

15. P. Attard, Langmuir 17, 4322 (2001).

16. Y. Y. Lin and C. Y. Hui, Journal of Polymer Science Part B: Polymer Physics 40, 772 (2002).

17. C. A. Miller and P. Neogi, Interfacial Phenomena. Marcel Dekker, Basel (1985).

18. A. M Schwartz, J. Colloid Interface Sci. 75, 404 (1980).

19. N. Amouroux and L. Léger, Langmuir 19, 1396 (2003).

20. P.-G. de Gennes, J. Phys. France 50, 2551 (1989).

21. E. Raphaël and P.-G. de Gennes, J. Phys. Chem. 96, 4002 (1992).

22. M. K. Chaudhury and G. M. Whitesides, Langmuir 7, 1013 (1991).

23. M. K. Chaudhury, J. Adhesion Sci. Technol. 7, 669 (1993).

24. A. N. Gent and P. Vondráček, J. Appl. Polym. Sci. 27, 4357 (1982).

25. P. Attard and J. L. Parker, Phys. Rev. A 46, 7959 (1992).

26. J. B. Pethica and A. P. Sutton, J. Vac. Sci. Technol. A 6, 2490 (1988).

27. J. R. Smith, G. Bozzolo, A. Banerjea and J. Ferrante, Phys. Rev. Lett. 63, 1269 (1989).

28. H. Hertz, J. Reine Angew. Math. 92, 156 (1882).

29. K. L. Johnson, K. Kendall and A. D. Roberts, Proc. R. Soc. Lond. A 324, 301 (1971).

30. J. A. Greenwood and J. B. P. Williamson, Proc. R. Soc. Lond. A 295, 300 (1966).

31. K. N. G. Fuller and D. Tabor, Proc. R. Soc. Lond. A 345, 327 (1975).

32. W. R. Chang, I. Etsion and D. B. Bogy, Trans. ASME: J. Trib. 109, 257 (1987).

33. S. K. R. Chowdhury and P. Ghosh, Wear 174, 9 (1994).

34. L. X. Zhang and Y.-P. Zhao, J. Adhesion Sci. Technol. 18, 715 (2004).

35. R. S. Sayles and T. R. Thomas, Nature 271, 431 (1978).

36. A. Majumdar and B. Bhushan, J. Tribol. 113, 1 (1991).

37. B. N. J. Persson and E. Tosatti, J. Chem. Phys. 115, 5597 (2001).

38. D. Maugis, J. Adhesion Sci. Technol. 10, 161 (1996). 